


d

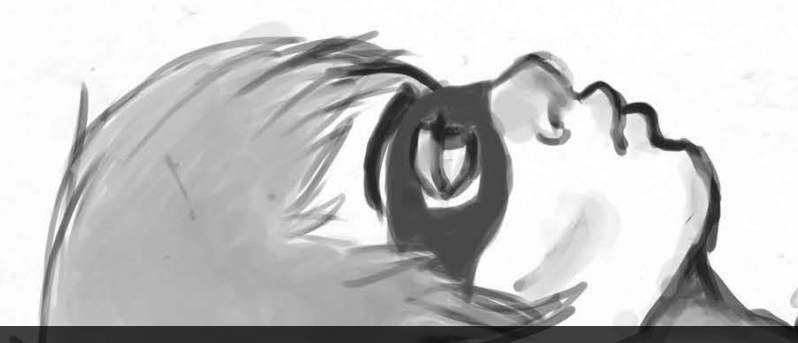

Diseño de un modelo para la evaluación de las competencias comunicativa, tecnológica e investigativa, en la modalidad de educación virtual de la Institución Úniversitaria Politécnico Grancolombiano 


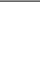




\title{
Diseño de un modelo para la evaluación de las competencias comunicativa, tecnológica e investigativa, en la modalidad de educación virtual de la Institución Universitaria Politécnico Grancolombiano ${ }^{1}$
}

Fecha de recepción: 13 de diciembre de 2010

Fecha de aceptación: 6 de abril de 2011

\author{
María Del Socorro Guzmán Serna \\ Politécnico Grancolombiano \\ mdsguzman@poligran.edu.co
}

Licenciada de la Universidad del Cauca,

Especialista en Ambientes virtuales de aprendizaje

(oEA-Virtual Educa) y Magíster en Educación de

la Universidad Pedagógica Nacional. Integrante

de los grupos de investigación Gestión vital

y Educación. Actualmente, es la Directora

académica de educación virtual del Politécnico

Grancolombiano.

\section{Resumen}

En estas ultimas decadas, la tendencia de la educación superior se ha encaminado a promover y fomentar su proceso de enseñanza-aprendizaje o aprendizaje por competencias, con el propósito de acercar el mundo educativo al mundo productivo. Pasar de la enseñanza al aprendizaje, para el Politécnico Grancolombiano, en su modalidad virtual, ha implicado repensar el currículo y con él, el ser y hacer de las prácticas educativas enfocadas al logro de competencias.

Desde esta perspectiva, el currículo y las prácticas se vienen enfocando en el estudiante, el perfil, las demandas y las posibilidades profesionales que le permitan poder desempeñarse como un profesional capaz de asumir los retos desde el ámbito profesional y personal.

\section{Abstract}

The tendency of higher education in recent decades has aimed to promote and enhance their teachinglearning process or learning skills, with the aim of bringing the world of education to the productive world. The shift from teaching to learning for Politécnico Grancolombiano, in its virtual education, has implied to re-think the curriculum as well as the being and doing of educational practices focused on the achievement of competencies.

From this perspective, the curriculum and practices are being focused on the student profile, the demands, and the professional opportunities that will enable professionals to serve as a qualified person able to meet the challenges from the professional and personal fields.

1 
Es importante destacar que el Politécnico Grancolombiano, en el área de educación virtual, se compromete con el plan de mejoramiento continuo desde la función investigativa de los sistemas de gestión de aprendizaje. En este proceso investigativo se asume el enfoque de la investigación acción-participativa, con el fin de visibilizar lo avanzado, promover el uso de las mejores prácticas y elaborar la propuesta de un modelo para evaluar las competencias.

\section{Palabras clave}

Educación virtual, competencias, competencias genéricas, evaluación, evaluación por competencias.
It is important to say that Politécnico Grancolombiano, in the area of virtual education, is committed to the plan of continuous improvement from the investigative function of learning management systems. In this investigative process, the approach of participatory action research is taken in order to visualize progress, promote the use of best practices, and develop a proposed model for assessing competence.

\section{Keywords}

Virtual Education, Skills, Generic Skills, Evaluation, Evaluation per Abilities. 


\section{Algunos Conceptos Básicos}

\section{Educación virtual}

Hacia finales de la década de los noventa la UNESCO (1998) definió la educación virtual como "entornos de aprendizaje que constituyen una forma totalmente nueva, en relación con la tecnología educativa (...) Un programa interactivo de carácter pedagógico que posee una capacidad de comunicación integrada”.

Benitez (2000) plantea que la educación virtual:

Debe estar cimentada en la construcción de conocimiento, debe poseer un sentido efectivamente comunicacional en la medida en que el aprendizaje es también proceso social, debe ser entendida como relación activa $y$ expresiva de los sujetos, utilizando para ello los medios a su alcance, no sólo las nuevas tecnologías (...) Comunicación que no se limita a trasmitir comunicación técnica, sino al despliegue de habilidades comunicacionales que implican observación, expresión, escucha, tolerancia, alteridad a través de actividades de expresión y argumentación no sólo escrita, sino con el uso de diversos lenguajes, para lo cual también las nuevas tecnologias son muy útiles.

Banet (2001) ve la educación virtual "como una combinación entre la tecnología de la realidad virtual, redes de comunicación y seres humanos".

Por su parte, Lara (2002) afirma que "la educación virtual es la modalidad educativa que eleva la calidad de la enseñanza-aprendizaje (...) que respeta su flexibilidad o disponibilidad (en cualquier momento, tiempo y espacio). Alcanza su apogeo con la tecnologia hasta integrar los tres métodos: asincrónica, sincrónica y autoformación”.

Para el Ministerio de Educación (MEN, 2010), en Colombia, la educación virtual:

Es una acción que busca propiciar espacios de formación, apoyándose en las TIC, para instaurar una nueva forma de enseñar y aprender (...) es una modalidad de la educación a distancia; implica una nueva visión de las exigencias del entorno económico, social, y politico, así como de las relaciones pedagógicas y de las TIC. No se trata simplemente de una forma singular de hacer llegar la información a lugares distantes, sino que es toda una perspectiva pedagógica.

Gómez (2009) la sume como "una modalidad educativa que apela al uso apropiado de las tecnologías de la información y la comunicación, TIC (internet, entre estas), como mediación y con un claro propósito educativo".

\section{Competencias}

Sobre la definición de este término se encuentran diversas posturas. En este aparte se han retomado definiciones más cercanas a la perspectiva de formación integral. Veamos algunas:

Jiménez (2007) manifiesta que desde la mirada de diversos teóricos las competencias son "un saber hacer contextualizado, que se fundamenta en una determinada acción, que actúa como impulso cognitivo para solucionar problemas, en la cual la mente humana elabora un conjunto de planes o aplica reglas generales de acción (heurísticas), en función de un contexto cultural, en el cual se desempeña".

Para Morales, mencionado por Jiménez (2007), "las competencias no se desarrollan con el mero saber, sino con el saber hacer o Know how o conocimientos procedimentales, que consisten en el dominio de reglas inferidas y construidas a partir de esquemas de procesamiento que permiten actuar inteligentemente en campos o dominios distintos".

Torrado (2000), por su parte, plantea que la competencia además de ser un saber, es un saber haciendo, apoyado en múltiples conocimientos que se van adquiriendo en el transcurso de la vida; también resalta que es la utilización flexible e inteligente de los conocimientos que poseemos lo que nos hace competentes frente a tareas específicas. 
Bogoya (2000) indica que trabajar en competencias implica pensar en la formación de ciudadanos idóneos para el mundo de la vida, quienes asumirán una actitud crítica ante cada situación, un análisis y una decisión responsable y libre y una idea de educación autónoma permanente, profundizando en aquellos aspectos que ellos mismos determinen.

El proyecto Tuning define las competencias como "una combinación dinámica de conocimiento, comprensión, capacidades y habilidades" y destaca que "una competencia es una capacidad que incluye habilidades, actitudes y valores. Esta debe contribuir a los resultados valorados por la sociedad y los individuos, ayudar a los individuos a satisfacer las demandas en una variedad de contextos y ser de importancia para los individuos y los especialistas".

\section{Las competencias genéricas}

Estas se definen como aquellas que son comunes a la gran mayoría de las profesiones y se relacionan con conocimientos, valores, rasgos de personalidad, aptitudes, aspectos requeridos por varias ocupaciones laborales o transferi-bles en actividades, sectores $u$ organizaciones.

El enfoque de competencias en el ámbito educativo, desde la perspectiva del Ministerio de Educación en Colombia, ha sido asumido como parte de las estrategias para garantizar la calidad académica y la articulación con los diferentes niveles educativos. Cabe destacar que desde el año 2008, el MEN definió como competencias genéricas a la comunicación en lengua materna y otra lengua internacional, pensamiento matemático, ciudadania, y ciencia, tecnología y manejo de la información, con las cuales pretende responder a:

Las necesidades del mundo globalizado, en el que las distancias se han acortado con los medios de comunicación y de transporte, las fronteras económicas y laborales tienden a difuminarse y la educación busca cada vez con más fuerza la internacionalización de los saberes, las profesiones, las titulaciones y los mercados de trabajo. Sin descuidar, por supuesto, la pertinencia frente al contexto local, para el cual estas competencias también constituyen una fuente de desarrollo, de modo que su implementación contribuirá al mejoramiento de la calidad de la formación y a su proyección internacional.

La pertinencia y la calidad de la educación superior dependen en gran medida de la implementación de cuatro competencias genéricas que permiten aprender durante toda la vida, formando profesionales capaces de moverse con destreza en el mundo globalizado.

\section{Evaluación}

Tenbrink (2002), en su libro Evaluación: guía práctica para profesores, recoge el planteamiento de Tyler sobre evaluación educacional que consistente en:

\begin{abstract}
(...) la comparación entre objetivos y resultados, programación por objetivos aún vigente (...), para ello se deben marcar objetivos concretos $y$ expresados en términos de conductas observables, en evidencias (...) La evaluación educativa se basa en una comparación minuciosa entre lo que se pretende conseguir y lo que realmente se consigue, parte de la actuación y la necesidad de retroalimentación para rectificar dirección de procesos y componentes (contenidos, estrategias).
\end{abstract}

De igual manera, Tenbrink retoma a Stufflebeam yShinkfiel(1985), quienes resaltan el movimiento de recontextualización de los años sesenta, referente al término evaluación planteado por Cronbach, Eisner, Stufflebeam y Stake, quienes trazaron un modelo de evaluación formativa y criterial. La evaluación pasa a definirse como "enjuiciamiento sistemático del valor o mérito de un objeto". Para Tenbrink, la evaluación no sólo implica al estudiante y su rendimiento, también implica al docente, los proceso de enseñanzaaprendizaje, los recursos y la institución.

Para Scriven (1967) la evaluación

(...) es una estimación o constatación del valor de la enseñanza, considerada no sólo en sus resultados, sino 
también en su proceso de desarrollo. La evaluación sumativa se centra en el estudio de los resultados, mientras que la evaluación formativa constituye una estimación de la realización de la enseñanza y contiene en sí el importante valor de poder servir para su perfeccionamiento al facilitar la toma de decisiones durante la realización del proceso didáctico.

Por su parte, McDonald (1971) considera que "la evaluación deber ser holística, es decir, tomar en consideración todos los posibles componentes de la enseñanza: procesos, resultados, contexto". Así mismo, considera que la enseñanza adquiere unas determinadas características distintas en cada situación, "por lo que es necesario acercarse desde una perspectiva ecológica y contextual de la evaluación misma".

Tyler (1973) define la evaluación como "una constante comparación de los resultados de aprendizaje de los alumnos con los objetivos previamente determinados en la programación de la enseñanza”.

Niño y otros (1995) mencionan a Elliot Eisner, Parlett \& Hamilton y Alvarado \& Sandoval como ejemplo del enfoque de la evaluación a modo de "una práctica pedagógica, comunicativa, intencionada, holística y cualitativa que responda a la demanda por la integración de intereses técnicos, interpretativos y emancipatorios; que corresponda pues al espíritu de la nueva Ley General de Educación y a las demandas que la sociedad hace a la educación".

Díaz (2010) asume la evaluación como "el proceso de obtener información y usarla para formar juicios que a su vez se utilizarán en la toma de decisiones".

\section{Evaluación por competencias}

La evaluación por competencias se ha considerado el paso fundamental de un proceso de aprendizaje. María Cristina Torrado (2000) propone un acercamiento entre competencia y logro: "podemos entonces afirmar que los indicadores de logro se refieren a la actuación, esto es, al uso de un conocimiento (...) El logro no es otra cosa que el conocimiento que se usa, es decir la competencia. Sobra decir que una competencia puede ser observada en múltiples indicadores".

En los planteamientos de Torrado la competencia tiene sinónimos tales como: Logro, capacidad, disposición, evidencia, reali-zación, función, demostración; cualquiera sea el enfoque sobre por qué, para qué, qué, cómo, cuándo y quién evaluar el aprendizaje y desarrollo de una competencia. Está claro que la competencia no es visible y que por eso se deben desarrollar estrategias para ver como se muestra, para conocer sus manifestaciones, evidencias, realizaciones o logros, lo que también es claro es que si es evaluable. La competencia supone conocimientos, saberes, valores, actitudes y habilidades que emergen de la interacción que se establece entre el individuo y su contexto, y que no siempre están dados de antemano.

Para Galo Clavijo (2008) la evaluación por competencias implica dar respuestas claras y concretas a preguntas como: ¿qué evaluar?, ¿cuándo evaluar?, ¿quién evalúa? y ¿cómo evaluar? Igualmente, requiere definir los instrumentos de evaluación, hacer revisión permanente y crítica de cómo se hace el proceso y cómo se incorporan los instrumentos teóricos prácticos para evidenciar las competencias. Indica que la evaluación debe ser el resultado de un diseño previo, un análisis riguroso de evidencias y criterios de evaluación.

\section{Avances en evaluación de competencias en educación virtual del Politécnico Grancolombiano}

En cuanto al tema de evaluación por competencias, el modelo de educación virtual del Politécnico Grancolombiano ya cuenta con varios avances, entre ellos están:

1. Planeación y planificación por objetivos de aprendizaje/competencias. La estructura de los programas y cada módulo cuenta con esta planeación y planificación por objetivos de aprendizaje/ competencias.

2. El modelo de educación virtual del Politécnico Grancolombiano, asume las competencias así: 


Competencias

Hacen referencia al perfil académico - profesional.

Tienen un rango de generalidad, más allá de la planificación.

Delimitan el tipo de profesional que se desea formar.

Se sitúan en el límite entre el final de la formación y el comienzo de la actividad profesional.

Engloban todas las dimensiones de la persona. Tratan de formar ciudadanos críticos y profesionales competentes.

El currículo tiende a una mayor interrelación e interdisciplinariedad.

Requiere una actitud distinta de los estudiantes con una mayor implicación y compromiso y una mayor responsabilidad en su propio aprendizaje que se fundamenta en su mayor autonomía.

El tutor se convierte en un asesor que apoya, ayuda, informa, entrena y supervisa a cada estudiante en su aprendizaje, respetando su estilo de aprendizaje y basándose en sus aprendizajes previos.

El tutor pasa a ser organizador y facilitador del aprendizaje.

3. La evaluación se considera un proceso de retroalimentación que determina idoneidad en los aprendizajes de acuerdo con las competencias. Para su evaluación se plantean criterios que le permitan al estudiante identificar para qué, por qué y cómo es la evaluación. El modelo de educación virtual le propone al estudiante actividades de aprendizaje que lo retan a la búsqueda y que lo enfrentan a escenarios reales.
En este proceso se hace evaluación tanto cuantitativa como cualitativa, lo cualitativo pretende de manera progresiva conocer los logros que van teniendo los estudiantes en cada módulo y con ellos en su programa. Referente a lo cuantitativo, los logros corresponden a una escala numérica, la cual también determina sus grados de avances.

4. Planeación de evaluación de competencias en los módulos. En la planeación de los módulos se han incluido: evaluación diagnóstica, evaluación de procesos y evaluación final, considerando en este proceso la autoevaluación, la coevaluación y la heteroevaluación de los aprendizajes.

Por otra parte, es de resaltar que cada proceso evaluativo se articula en una estrategia didáctica. De la misma manera, se tiene planeado el proceso evaluativo: cómo, cuándo y con qué recursos; en cada módulo se especifican los productos y las competencias que se deben lograr.

Los módulos cuentan con pruebas escritas, proyectos, foros, wikis, blogs y redes.

A través de los proyectos, foros, talleres, casos $\mathrm{y}$ evaluaciones, el estudiante recibe retroalimentación de avances, de aspectos por mejorar $\mathrm{y}$ aspectos por alcanzar, con indicaciones puntuales de cómo hacerlo.

5. Redefinición de funciones. En este proceso, los docentes vienen asumiendo el rol de autores, tutores y expositores, roles que les exigen cambios en sus prácticas. Desde estos roles se enfocan al diseño y planificación, gestión del aprendizaje, evaluación, revisión y mejora, y trabajo en equipo de manera colegiada; esta última permite y facilita el cambio.

\subsection{Actividades de evaluación bajo el enfoque de competencias}

Definir las actividades de evaluación en el enfoque de competencias también requiere cambiar la forma de realizar algunas de estas actividades que se vienen utilizando en la evaluación tradicional. El procedimiento que se ha ejecutado en educación virtual a la fecha es: 
- Decidir la información que se recoge para la evaluación y especificar cómo y cuándo.

- Seleccionar y elaborar, en cada caso, los instrumentos necesarios para la recogida y registro de la información.

- Preparar, de forma explícita y diferenciada, la información sobre el sistema de evaluación y su proceso.

- Recoger la información.

- Valorar la información recogida.

- Decidir la calificación.

- Comunicar el resultado de la evaluación a los estudiantes.

- Atender las consultas de los estudiantes sobre la evaluación.

- Determinar las oportunidades de revisión, mejora y superación.

- Sistematizar los resultados de la evaluación para posibilitar el análisis y reflexión en torno a los mismos.

Así mismo, se vienen incorporando otras actividades para evaluar competencias, tales como:

Verificar el sistema de evaluación y calificación que se seguirá de acuerdo con un proceso de evaluación inicial, continua y final y su adecuación a la programación académica o de módulo.

- Evidenciar la coherencia entre el desarrollo del módulo y la evaluación.

- Desarrollar estrategias para facilitar la autoevaluación y la evaluación conjunta con otros colegas, a nivel de módulo.

- Compartir la información recogida con los estudiantes, teniendo en cuenta sus apreciaciones.

- Ayudar a realizar una evaluación personal de lo que realmente ha aprendido el alumno y de su compromiso como estudiante que ejerce la autocrítica.
Falta por implementar:

- La previsión de posibles fracasos de estudiantes en el avance de su proceso de aprendizaje y forma de recuperación.

- Establecer fórmulas de aprovechamiento del aprendizaje de los estudiantes en cada semestre o módulo.

- Elaborar instrumentos de chequeo, observación y registro de aprendizajes.

- Elaborar para todos los programas la matriz de competencias determinando nivel de logro e indicadores.

\subsection{Sílabo y guía de aprendizaje del estudiante}

El sílabo de los módulos que conforman el plan de estudio es el documento imprescindible y orientador que tiene la Institución. En este documento se debe reflejar la apuesta de la Institución, en términos de aprendizaje por competencias, pensado desde el estudiante. Es de resaltar, que si bien cada programa y cada módulo cuentan con su sílabo, es necesario que los mismos sean revisados con estos requerimientos.

6. A partir del segundo semestre del 2010, por política Institucional, se implementó el proceso de caracterización, el cual exige que todos los estudiantes que ingresan a la universidad, en cualquiera de sus modalidades y antes de dar inicio a su semestre, deben ser valorados en lectoescritura, matemáticas e inglés y deben recibir inducción en tecnología. Los resultados de las valoraciones serán el punto de partida para el diseño de propuestas que contribuyan al mejoramiento continuo de las competencias.

Respecto a la competencia investigativa, los programas de educación virtual a partir del 2011 iniciarán la segunda fase, correspondiente a proyectos articulados por áreas. Esta fase pretende reforzar y potenciar los semilleros de investigación y avanzar en propuestas de articulación con la tercera fase correspondiente a la investigación aplicada.

De igual manera, a partir del 2011, se implementará el proceso de seguimiento y contraste de resultados de las prácticas y exámenes ECAES. 


\section{Propuesta para la evaluación de las competencias comunicativa, tecnológica e investigativa}

La propuesta se sustenta en el modelo de educación virtual y busca, desde el mismo, articular tres niveles de evaluación. Un primer nivel, desde la estructura de contenidos y ambientes de aprendizaje propios del modelo de educación virtual; un segundo nivel desde el seguimiento a través de la carrera, aplicando valoraciones en tres momentos: prueba de entrada, prueba a mitad de carrera y prueba al finalizar carrera; y un tercer nivel desde la triangulación de resultados de las pruebas de seguimiento de carrera, con resultados de la práctica aplicada y resultados del ECAES.

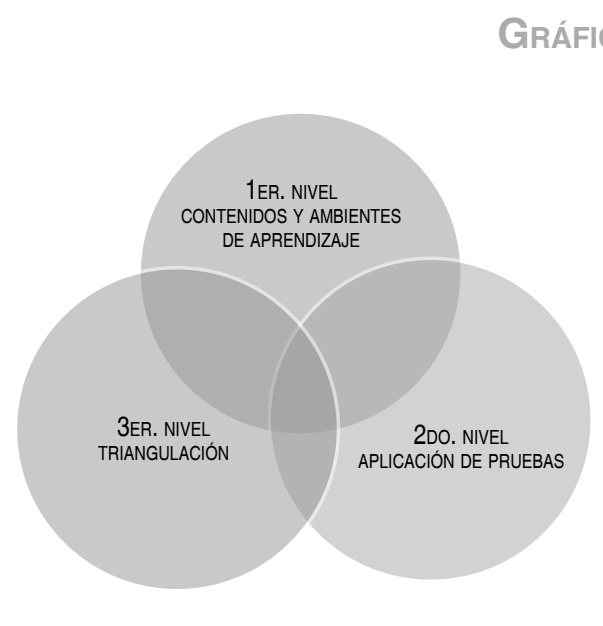

Fuente: María del Socorro Guzmán Serna.

La importancia de evaluar el proceso desde la estructura de contenidos y ambientes de aprendizaje radica en poder dar cuenta, a través del mismo modelo, de cómo se estructuran y desarrollan los procesos para que el estudiante adquiera la competencia.

Valorar en tres momentos a los estudiantes, permite conocer los avances en los niveles de cada competencia y a su vez autoevaluar los procesos y estrategias definidas por la Institución para este logro.

Y finalmente, triangular resultados de valoraciones académicas con las prácticas y resultados de pruebas ECAES permite conocer logros, alcances, fortalezas y debilidades que aportan al mejoramiento continuo del proyecto de formación institucional.

Primer nivel: desde la estructura de contenidos y ambientes de aprendizaje, propios del modelo de educación virtual.

\section{Algunas consideraciones}

En primer lugar, es indispensable asumir la evaluación como parte integral del currículo, la cual dinamiza y facilita el mejoramiento continuo en el aprendizaje, desde esta perspectiva la evaluación es formativa. En segundo lugar, la evaluación, al ser parte del currículo, no sólo se debe planificar sino que se debe desarrollar como un elemento fundamental en el proceso de aprendizaje para estimar logros y resultados. Y en tercer lugar, se debe tener presente que la evaluación siempre afecta tanto resultados como procesos y actores, por lo tanto, debe ser permanente y continua.

Por otra parte, el aprendizaje basado en competencias, requiere tener claras las competencias que se desea que adquieran los estudiantes y analizar el modo más efectivo para desarrollarlas. Supone un enriquecimiento de las metodologías de aprendizaje, un mayor seguimiento y tutoría de manera individual y grupal, y toda una gama de técnicas de evaluación de los aprendizajes.

El tutor, al modificar su rol en el proceso de aprendizaje, debe enfocarse en la organización, seguimiento y evaluación del aprendizaje de los estudiantes.

La formulación de las competencias ha de tener en cuenta no sólo los contenidos en términos de conocimientos y de aplicación (saber y saber hacer), sino también la integración de aspectos referidos a actitudes y valores que se aplican a la solución de problemas y conducen a la obtención de resultados en un contexto académico-profesional. La evaluación se hará en función de indicadores de resultados que se tendrán en cuenta a lo largo del proceso del aprendizaje del estudiante.

Evaluar las tres competencias genéricas representadas en la gráfica implica tener presente el aporte que se le hace al perfil académico-profesional. 


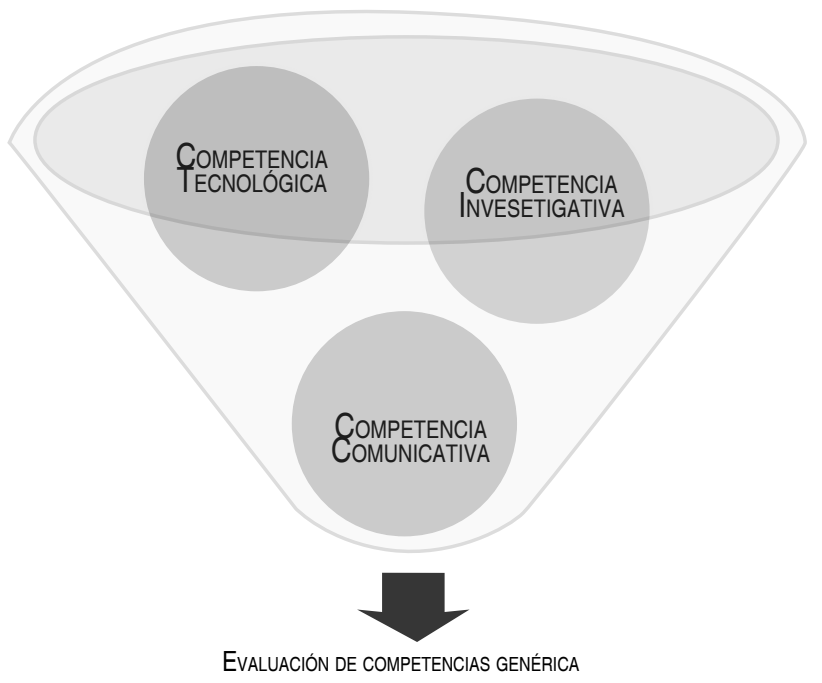

Fuente: María del Socorro Guzmán Serna.

Para su planificación en cada módulo, se propone que los tutores asuman el proceso en la gráfica 3 .

El proceso implica desarrollar los siguientes pasos:

1. Tener presente el perfil profesional y las tres competencias genéricas (comunicativa, tecnológica, investigativa).

2. Formular los alcances en términos de resultados de aprendizaje, desde la perspectiva del estudiante.

3. Determinar los conocimientos, habilidades, comportamientos o actitudes y valores que el estudiante debe adquirir para desarrollar la competencia tal como ha sido formulada.

4. Definir y describir las estrategias de aprendizaje a emplear para desarrollar los contenidos de acuerdo con el sentido de la competencia.

5. Realizar el plan evaluativo acorde con un cronograma académico y un modelo de educación virtual y planear los siguientes aspectos:

6. Tiempo de realización que requiere cada actividad por parte del estudiante de acuerdo a los créditos académicos.

- Seleccionar los indicadores para cada competencia.

- Seleccionar las herramientas de evaluación que le permitan medir de forma cuantitativa y cualitativa los indicadores elegidos.

- Dar valor (ponderación) que tendrá cada competencia en el módulo.

- Definir los criterios de evaluación de cada competencia.

- Elaborar un informe-resumen que indique el proceso y la valoración, así como sugerencias de ajustes o mejoramiento.

- Es necesario que los tutores que manejan un mismo módulo, realicen esta planificación conjunta.

En cada uno de estos aspectos se debe tener en cuenta el modelo de educación virtual y sus avances en términos de ponderación y actividades. 
GráFICA 3. PROCESO de PLANIFICACIÓn PARA MÓdULOS PROCESO
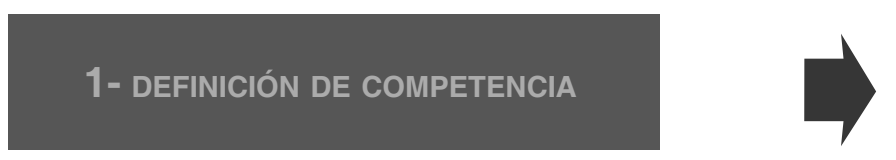

2 - Formullción de Resultados de APRENDIZAJE POR COMPETENCIA

\section{4 - Definición DE etrategias dE} APRENDIZAJE

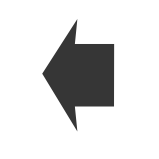

3 - Determinación de CONOCIMIENTO, HABILIDADES, ACTITUDES Y VALORES
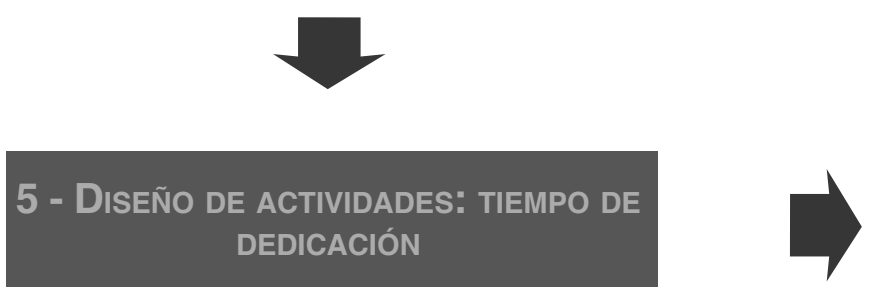

\section{6 - SistemA DE EVALUACION: INDICADORES Y TÉCNICAS.}

Fuente: María del Socorro Guzmán Serna.

El segundo aspecto a realizar después de la planificación es la guía de aprendizaje del estudiante.

\section{Estructura}

Para el desarrollo de la estructura se propone concebir la competencia como saber, habilidad, destreza y valores.

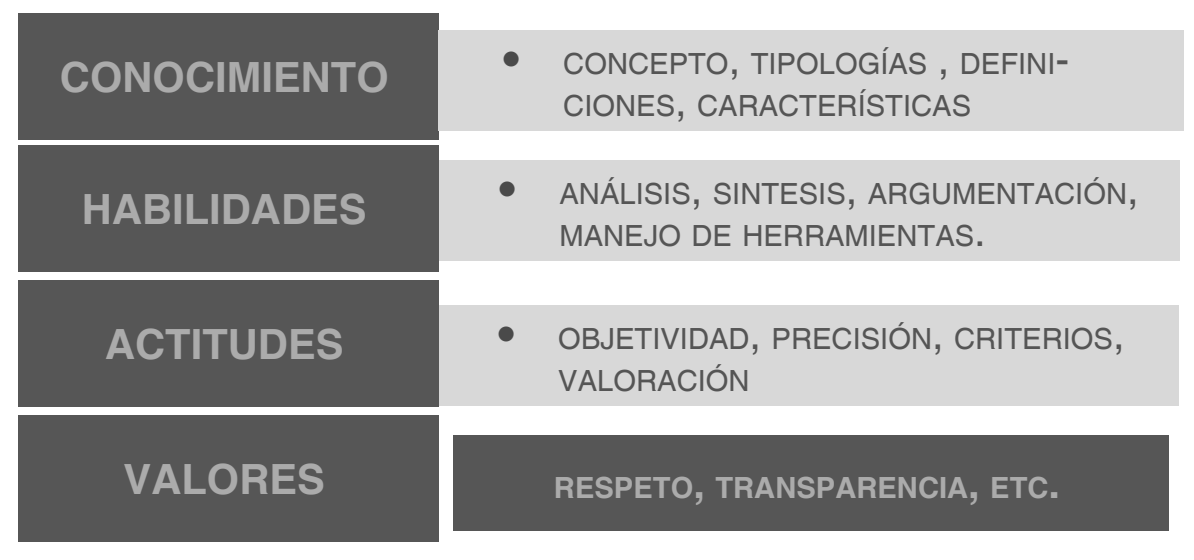

Fuente: María del Socorro Guzmán Serna 
La estructura contempla los siguientes aspectos:

Gráfica 5. Estructura

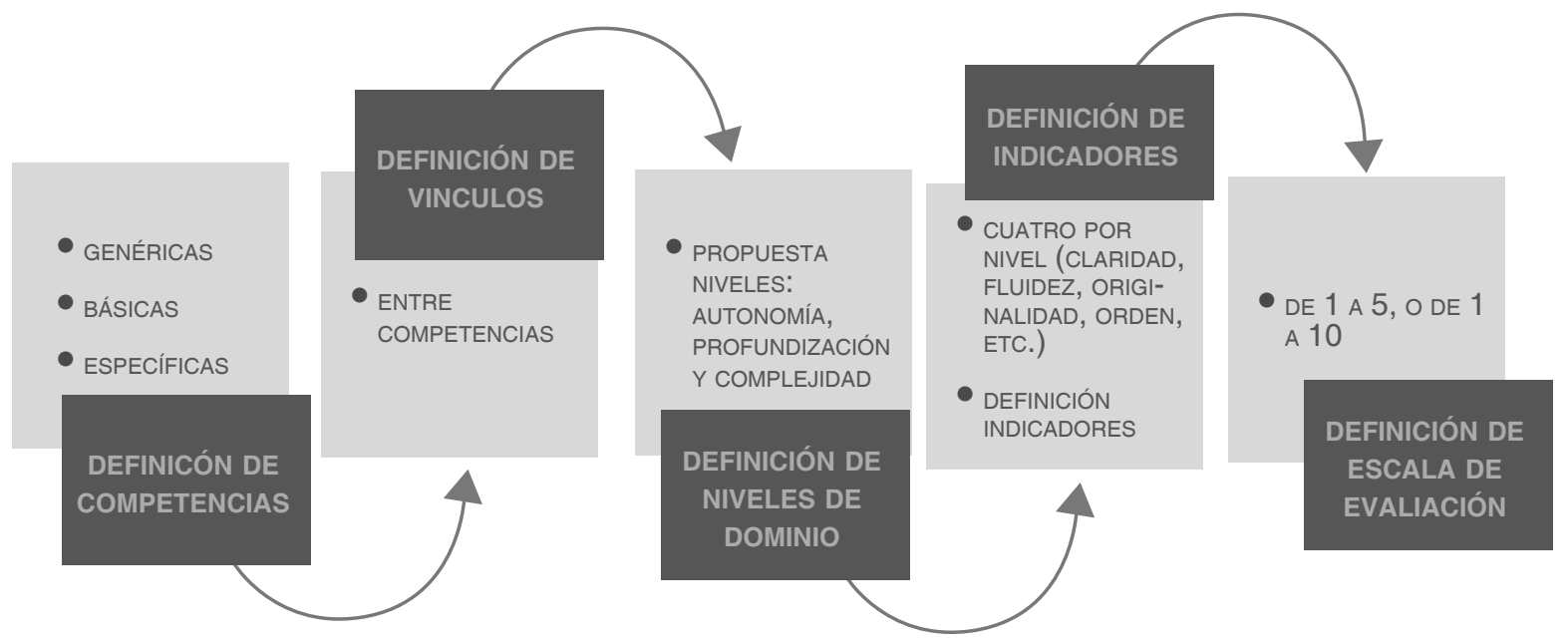

Fuente: María del Socorro Guzmán Serna.

Una vez definidas las competencias, se buscan los vínculos que existen entre ellas y se establecen los niveles de dominio. Para los niveles de dominio se propone esta matriz basada en los siguientes criterios:

1. Ser competente requiere un manejo de conocimiento que puede ser mayor o menor en función de la necesidad requerida para resolver un problema o acción concreta.
2. La competencia implica un nivel de autonomía personal, podriamos decir que a mayor dependencia menor autonomía.

3. En razón a que la competencia debe ser demostrada en un contexto o situación en la que se debe ejercer, la complejidad se convierte en un elemento fundamental para determinar la apropiación.

TABLA 1.

\section{Nivel de dominio Autonomía}

Nivel 1

Necesita orientación y supervisión.
A partir de las normas y criterios básicos demuestra un dominio de los contenidos.

\section{Profundización Complejidad}

Asume situaciones sencillas y resuelve problemas en contextos estructurados.
Nivel 2
Asume riesgos y toma decisiones en el contexto de situaciones nuevas.
Selecciona la información más importante, de forma sistemática y fluida aplicándola con eficiencia. Desarrolla todo el proceso.
Interviene en situaciones menos estructuradas y de creciente complejidad. 


\begin{tabular}{|c|c|c|c|}
\hline Nivel de dominio & Autonomía & Profundización & Complejidad \\
\hline Nivel 3 & $\begin{array}{l}\text { Asume riesgos y } \\
\text { emprende actua- } \\
\text { ciones con total } \\
\text { independencia. }\end{array}$ & $\begin{array}{l}\text { Anticipa, planifica y } \\
\text { diseña, de manera } \\
\text { creativa, respuestas } \\
\text { y soluciones a situa- } \\
\text { ciones complejas. }\end{array}$ & $\begin{array}{l}\text { Se desenvuelve } \\
\text { en situaciones } \\
\text { complejas, y halla } \\
\text { soluciones inte- } \\
\text { grales y globales. } \\
\text { Tiene en cuenta las } \\
\text { interrelaciones y } \\
\text { la transferibilidad } \\
\text { de las posibles } \\
\text { soluciones. }\end{array}$ \\
\hline
\end{tabular}

Fuente: María del Socorro Guzmán Serna.

\section{Sugerencias de técnicas}

Existen varias técnicas a aplicar. Para el modelo de educación virtual se sugieren las siguientes: pruebas de respuesta larga, pruebas de respuesta corta, pruebas objetivas, pruebas con preguntas abiertas, tipos de pensamiento, técnicas de observación, pruebas de autoevaluación, escala de actitudes, portafolio, informe, pruebas de ejecución, trabajo de prácticas, solución de problemas, mapa conceptual, debate, resolución de casos, proyectos y ensayos, diarios.

TABla 2. EJemplo de VAloraciones POR tÉCNICA

Técnica

Conocimiento

Habilidad

Actitud

\begin{tabular}{cccc}
\hline Solución de problemas & $\mathrm{xx}$ & $\mathrm{xx}$ & $\mathrm{xx}$ \\
\hline $\begin{array}{c}\text { Proyecto de investig- } \\
\text { ación formativa aplicada }\end{array}$ & $\mathrm{xx}$ & $\mathrm{xx}$ & $\mathrm{x}$ \\
\hline Resolución de casos & $\mathrm{xx}$ & $\mathrm{xx}$ & $\mathrm{xx}$ \\
\hline Foro /Debate & $\mathrm{x}$ & $\mathrm{xx}$ & $\mathrm{xx}$ \\
\hline Ensayo, articulo & $\mathrm{xx}$ & $\mathrm{xx}$
\end{tabular}

Fuente: María del Socorro Guzmán Serna. 
Una vez definido todo el proceso, es necesario reflexionar sobre la importancia de la evaluación. Si retomamos a Rotger (1992) la evaluación debe cumplir con cinco elementos:

1. Debe ser útil, es decir debe atender a las necesidades del estudiante. Cobra sentido hacerse las siguientes preguntas: ¿vale la pena realizar la evaluación?, ¿satisface las necesidades reales?

2. Debe ser viable, es decir realista. Cobra sentido cuando se obtiene información que permite hacer conclusiones que conlleven a mejorar los resultados.

3. Debe ser precisa, es decir los resultados deben ser objetivos y confiables; para ello los instrumentos y técnicas utilizados deben ser válidos y precisos con el fin de recoger la información requerida para el análisis, por lo tanto se necesitan resultados claros y comprensibles.

4. Debe ser transparente, es decir que se debe contar con toda la información de manera precisa y rigurosa para garantizar esa transparencia.

5. Debe ser justa, es decir que debe ser un proceso ético.

\section{Flexibilización de la evaluación}

En la modalidad virtual prima el aprendizaje autónomo, por ello la evaluación y sus procesos deben ser flexibles desde:

\section{- Estrategias}

- Técnicas

- Actores

- Momentos de realización

Por ello es fundamental el acompañamiento al estudiante en el ámbito individual y grupal, teniendo en cuenta que reciba información a tiempo (feedback inmediato) sobre la valoración que se hace en torno a sus avances.
La evaluación deja de ser sólo un juicio de valor, una nota, para convertirse en un indicador del estado del aprendizaje del estudiante y sus logros. El juicio de valor que recibirá al final (la nota), estará ajustado al trabajo que él mismo sabe que ha realizado.

\section{Importancia de la tutoria}

Una eficaz labor de evaluación exige el protagonismo del trabajo de tutoría. El acompañamiento es una nueva función que puede enfatizar la labor del tutor, asumiendo un rol más simétrico con el estudiante.

El tutor, con esta nueva función, planifica su trabajo y "ayuda" a planificar el trabajo del estudiante (aprendizaje); contribuye a encontrar sentido a los contenidos con el contexto experiencial (background) del estudiante; le hace reflexionar sobre los conocimientos e intencionalidad de las competencias a desarrollar en el módulo; proporciona recursos para que el estudiante los integre con otros que buscará en diversas fuentes; orienta el sentido de los conocimientos y experiencias que va adquiriendo el estudiante para que genere los propios; facilita la creación de una "cultura" colegiada de aula en la que se compartan intereses, conocimientos, pensamientos, experiencias; participa en la moderación de los debates; sigue y apoya el proceso de aprendizaje del estudiante; evalúa y orienta en ese proceso.

Sin una labor en este sentido, es muy poco probable que se dé el desarrollo y evaluación de competencias y, por tanto, no se avanza en el camino adecuado que exige el proceso de innovación educativa propuesto.

Segundo nivel: Desde el seguimiento a través de la carrera, aplicando valoraciones en tres momentos: prueba de entrada, prueba a mitad de carrera y prueba al finalizar carrera.

Gráfica 6. Tiempos
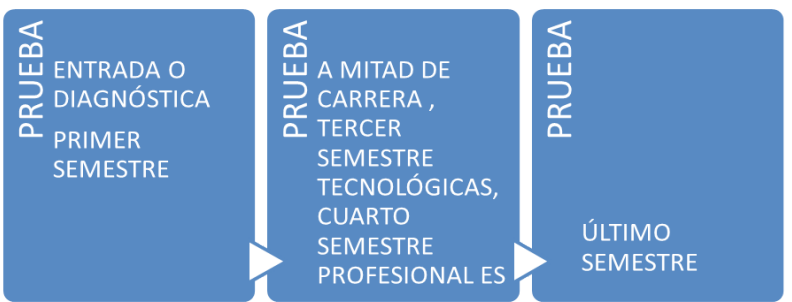

Fuente: María del Socorro Guzmán Serna. 
Al respecto, a partir del segundo semestre del 2010, la Vicerrectoría Académica instituyó la aplicación de pruebas de caracterización, las cuales incluyen una prueba de entrada en las áreas de lectoescritura, matemáticas e inglés, con el propósito de hacer un diagnóstico de entrada de los estudiantes; es de resaltar que dichas pruebas están elaboradas por nivel de competencias.

Así mismo, se definió hacer seguimiento durante el semestre, realizar contrastes con las pruebas diagnósticas y continuar el proceso de seguimiento a través de una segunda prueba a mitad de carrera y realizar una tercera valoración al finalizar la carrera. Este proceso pretende buscar y diseñar alternativas de acompañamiento y planes de mejoramiento continuo.

Tercer Nivel: Triangulación de resultados de las pruebas de seguimiento de carrera con resultados de la práctica aplicada y resultados ECAES.

\section{Gráfica 7. Triangulación de Resultados}
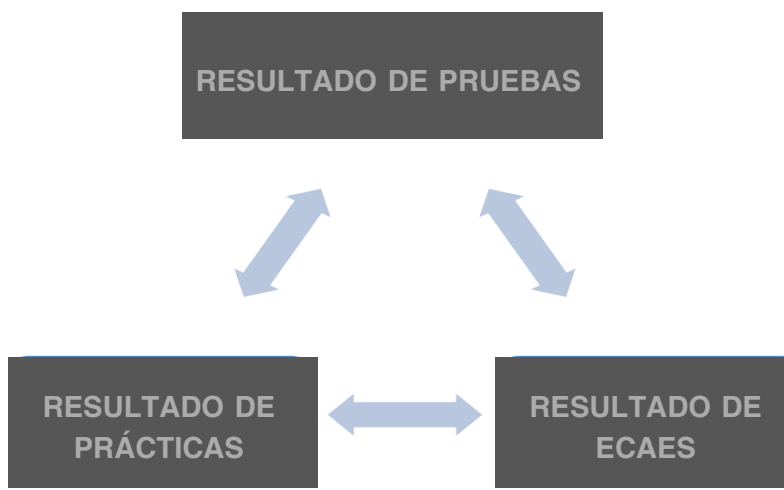

Fuente: María del Socorro Guzmán Serna.

Desde la Vicerrectoría Académica, se estableció como plan estructural, además del segundo nivel, realizar un proceso de contraste y triangulación de resultados cognitivos, con resultados de la práctica aplicada y resultados de pruebas ECAES.

Este plan tiene como propósito dar cuenta de procesos, estrategias y resultados implementados para indicar el manejo de un currículo por competencias.

\section{Conclusiones}

Respecto a las competencias, podemos decir que dar cuenta de ellas en el marco de la educación virtual no es sencillo, implica estructurar el currículo, los módulos, las actividades académicas y las intervenciones tanto del tutor como de los estudiantes, de tal manera que se pueda ir valorando la adquisición o no de estas competencias para hacer los ajustes pertinentes y lograr el cometido.

El reto del ejercicio académico es poder estructurar y combinar estrategias, mediaciones y herramientas que posibiliten el desarrollo de estas competencias.

Dado que la competencia se identifica con el concepto de "capacidad" que posee un estudiante y esto se verifica en la práctica, en la interacción con el contexto, se hace necesario describir los contenidos requeridos para el desarrollo de las mismas.

Desde el saberes preciso que los estudiantes den cuenta y manejen conocimientos, conceptos, definiciones, datos, hechos e informaciones. Desde el saber hacer se requiere que den cuenta de las diversas formas de transferir el saber, habilidades, destrezas, técnicas y procesos que aplican. Desde el saber ser la actitud, los valores, la responsabilidad, la autonomia, el compromiso, el interés y el respeto son determinantes. En el saber estar es indispensable la disposición a la comunicación e interacción interpersonal y la disposición al trabajo colaborativo. Y desde el saber emprender, se requiere predisposición a la creatividad, la innovación y la creación.

Respecto a las competencias genéricas podemos decir que aportan al desarrollo de otras habilidades tales como:

- Habilidades sociales. Se pueden apreciar en la interacción del trabajo en grupo colaborativo. La relación con sus compañeros les da identidad, les permite tratar con diversas personalidades, generar empatía $\mathrm{y}$ construir capital social. 
- Habilidades para la resolución de conflictos. La interacción y construcción en colectivo requiere tener en cuenta los intereses y necesidades de los otros, identificar y analizar manejo de poder, dar reconocimiento, establecer reglas para el desarrollo y compromisos académicos, establecer prioridades y decidir renuncias y circunstancias lo cual conlleva a acuerdos y manejo constructivo de las diferencias.

También podemos decir que las competencias genéricas contribuyen $\mathrm{y}$ permiten establecer diversas formas de relacionarse con los demás, cooperar y trabajar en equipo, manejar y resolver conflictos. Es decir permiten que las personas aprendan a vivir y trabajar con otros. De igual manera, permiten crear identidad personal, dinámica social de los roles que desempeñan y desean desempeñar y capacidad de traducir las necesidades y deseos en actos de voluntad: decisión, elección y acción.

Las competencias genéricas como ejes transversales son dinamizadoras y gestoras del desarrollo personal y profesional con un propósito claro de desarrollo y evaluación durante la carrera. Además de asumir la formación desde el conocimiento (saber), asume el ser, saber hacer, actuar y emprender. A partir de esta perspectiva, las competencias son una expresión concreta de los diversos recursos que pone en juego un estudiante cuando lleva a cabo una actividad, cuando hace uso o manejo de lo que debe hacer, de lo que sabe de manera integrada en diversos contextos y no del conocimiento aislado para dar cuenta de su desempeño, que es lo que busca la Institución. Por ello integra y activa conocimientos, habilidades, destrezas, actitudes y valores.

Para el Politécnico Grancolombiano el sentido de las competencias adquiere verdadera importancia cuando prevalecen las actitudes, motivaciones, valores, etc., además de los conocimientos, habilidades y destrezas

\section{De la evaluación es importante resaltar:}

1. Los cambios paradigmáticos generan resistencia tanto en los autores y tutores como en los estudiantes, resistencia que depende mucho del manejo que el autor y el tutor le den a los procesos cuando explican los beneficios para alcanzar cada logro.

2. Se requiere de formación tanto al autor como al tutor.

3. Es fundamental que el proceso en cada programa se realice de manera colegiada para evitar incoherencias y confusiones para los estudiantes.

4. Es fundamental que los tutores diferencien qué es evaluar por competencias y qué es calificar.

5. Se requiere experimentar, sistematizar y hacer seguimiento de las mejores prácticas para avanzar e implementar mejoras. 


\section{Referencias}

1. Banet, M. (2001). Paradoja de los Entornos virtuales. México: Editorial April.

2. Benítez, R. (2000). La educación virtual desafío para la construcción de culturas e identidades. Ponencia Congreso Proyección de la Interacción Latinoamericana en el siglo XXI. Mesa IV Políticas culturales e identidad latinoamericana. México.

3. Bogoya, M. (2000). Competencias y proyectos pedagógicos, Colombia. Resumido por la profesora Swapna Puni Estévez Singh, bachiller en ciencias. Consultada en junio 2010 de http:// panelsd.iespana.es/col000201.htm

4. Clavijo, G. (2008). Congreso Cartagena de Indias, septiembre.

5. Díaz, F. (2010). Modelo para autoevaluar la práctica docente. Madrid, visita novedades profesorado.

6. Flores, H. (2007). Proyecto Tuning, América Latina. Reflexiones y perspectivas de la educación superior en América Latina. Universidad Deusto, Universidad Groningen, Bilbao.

7. Gómez, O. (2009). Educación virtual Inclusiva. Antioquia: Universidad Católica del Norte. Consultada en agosto 2010 de http://www.colombiaaprende. edu.co/html/mediateca/1607/ articles-132491_archivo.pdf

8. Jiménez (2007). Neuropedagogía, lúdica y competencias. Bogotá: Editorial Magisterio.

9. Lara, L. (2002). ARA, Segundo Congreso Virtual Integración sin barreras en el siglo XXI, Red de integración especial, grupo 1. Ponencia Análisis de los recursos interactivos en las aulas virtuales. Argentina.
10. MacDonald, B. (1971). The

Evaluation of the Humanities Curriculum Project: A Holistic Approach. The theory into practice, pág. 163-167.

11. Ministerio de Educación Nacional, MEN. (2010). Consultada en agosto 2010 de http: //www.mineducacion.gov. co/1621/article-196492.html

12. Ministerio de Educación Nacional, MEN. (2009). Competencias genéricas en Educación Superior. Boletín Informativo No. 13. Diciembre-2009. Bogotá:

Panamericana.

13. Niño, L. et al. (1995). Evaluación, Proyecto educativo y descentralización educativa. Bogotá: Universidad Pedagógica Nacional. Posgrados en Evaluación Escolar y Desarrollo Educativo Regional.

14. Politécnico Grancolombiano. Historia de la Institución Tecnológica. Bogotá. Consultado en junio 2010 de http://www.poligran.edu.co/eContent/ newsdetail.asp?id=197\&idcompany=28 \&idscategory $=64$

15. Politécnico Grancolombiano. (2004). Proyecto Educativo Institucional PEI, Bogotá, (pág. 8).

16. Politécnico Grancolombiano. (2008). Visión. Bogotá. documento interno Planeación estratégica Michelsen P. (2008).

17. Politécnico Grancolombiano. (2008). Documento Interno de trabajo Institucional. Planeación estratégica 2008-2013. Bogotá.

18. Politécnico Grancolombiano. (2009). Modelo Institucional. Bogotá, documento interno.

19. Politécnico Grancolombiano. (2009). Modelo Comunicativo. Bogotá, documento interno. 
20. Politécnico Grancolombiano. (2008). Investigación Formativa. Bogotá, documento interno. Departamento de Investigación.

21. Politécnico Grancolombiano. Institución. Bogotá. Consultado en julio 2010 de http://www.poligran. edu.co/eContent/NewsDetail. asp?id=273\&IDCompany=28

22. Politécnico Grancolombiano. Filosofía Institucional. Misión. Bogotá. Consultado en junio 2010 de http://www. poligran.edu.co/eContent/newsdetail. asp?id=276\&idcompany=28\&idscategor $\mathrm{y}=65$ \&itemmenu=2_250\#

23. Rotger, B. (1992). Educación y futuro. Monografías para la reforma. Evaluación formativa. Madrid: Cincel.

24. Scriven, M. (1967). The methodology of evaluation. En Stake, R.E. Aera Monograph series curriculum evaluation. Chicago: Randa Mc Nally.

25. Tenbrink, T. (1984). Evaluación guía práctica para profesores. Madrid: Narcea.

26. Torrado, M. (2000). Educar para el desarrollo de las competencias: una propuesta para reflexionar. Resumido por la profesora Swapna Puni Estévez Singh del libro Competencias y Proyectos Pedagógicos. Bogotá: Universidad Nacional de Colombia. Consultado en agosto 2010 de http:// panelsd.iespana.es/col00020.htm

27. Torrado, M.C. (2000). Educar para el desarrollo de las competencias. Una propuesta para reflexionar. En Bogoya, D. et al. Competencia y proyecto pedagógico. Bogotá: Universidad Nacional de Colombia.

28. Tyler, R.W. (1973). Principios básicos del currículo. Buenos Aires: Troquel.
29. UNESCO (1998). Conferencia Mundial sobre la Educación Superior. La educación Superior en el siglo XXI, visión y acción, Paris (5-9 octubre de 1998). Tomo V, plenaria. 


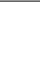

